\title{
Fuzzy Eigenvectors of Real Matrix
}

\author{
Zengfeng Tian (Corresponding author) \\ Composite Section, Junior College, Zhejiang Wanli University \\ Ningbo 315101, Zhejiang, China \\ Tel: 86-574-8835-7771Ｅ-mail: bbtianbb@126.com
}

\begin{abstract}
Real eigenvectors of real matrix is extended to fuzzy eigenvectors. The structure of fuzzy eigenspaces and relationships between real eigenspaces and fuzzy eigenspaces of real matrix are studied. Using fuzzy eigenvector, we give a sufficient condition to existence of solution of fuzzy linear systems.
\end{abstract}

Keywords: Fuzzy number, Eigenvector, Eigenvalue

\section{Introduction}

The real eigenvalues and eigenvectors of real matrix play a center role in mathematics and engineering. So are the fuzzy eigenvalues and eigenvectors. Fuzzy eigenvalues were first studied by Buckley (1990, p187-197) to analyze input-output of systems. Massa et al (2008, p63-85) determined both the fuzzy eigenvalues and eigenvectors of a finite element model defined with fuzzy parameters in their work. In Alevizos' work(2007, p704-721), fuzzy eigenvalue problem was converted to an ordinary one in order to study correspondence of fuzzy data. To produce a fuzzy eigenvector weight estimate, Wang and Chin (2006, p1257-1275) used the ordinary eigenvector through the solution of a linear programming model in their paper.

Fuzzy linear system (FLS for short) is an important fuzzy modal. The existence of solution of FLS is different from its real counterpart. As to FLS $A \tilde{x}=\tilde{y}$, where $A$ is a real matrix and $\tilde{x}, \tilde{y}$ are fuzzy vectors, whether there exist solutions depends on the choice of $\tilde{y}$, even if $A$ is invertible (Frideman, 2006, p1257-1275). However, there is no study on how to choose an appropriate $\tilde{y}$ such that there are solutions to $A \tilde{x}=\tilde{y}$. This work originates from this problem. For a real linear system $A x=y$, where $A$ is real matrix and $x, y$ are real vectors, it is obvious that if $y \in V_{\lambda_{1}}+V_{\lambda_{2}}+\cdots+V_{\lambda_{m}}$, where $\lambda_{i}$ is a nonzero eigenvalue and $V_{\lambda_{i}}$ is an eigenspace of $A$ then there exist solutions to $A x=y$. We will prove this result holds for FLS. In other words, we give a sufficient condition of existence of solutions to FLS by using fuzzy eigenspaces.

The structure of this paper is organized as follows. In Section 2, we introduce some definitions, results on fuzzy vector space and the embedding approach proposed by Friedman et al (Frideman, 2006, p1257-1275) that is used to transform a fuzzy linear system into an equivalent crisp function linear system. Fuzzy eigenvectors of real matrix is concerned in Section 3 followed by the structure of fuzzy eigenspaces in Section 4. Relationship between the fuzzy eigenvectors and real ones is discussed in Section 5. As an application of fuzzy eigenvectors, a sufficient condition to existence of solution of fuzzy linear system is given in Section 6, and the concluding remarks are related in Section 7.

\section{Preliminaries}

In this section we recall the basic notations of fuzzy number arithmetic, real eigenvectors of real matrix and fuzzy linear system.

Definition 1. (Ma, 2000, p55-58) A fuzzy number is a fuzzy set $\tilde{u}: \mathfrak{R} \rightarrow I=[0,1]$ which satisfies

- $\tilde{u}$ is upper semicontinuous.

- $\tilde{u}(x)=0$ outside some interval $[c, d]$.

- There are two real numbers $a, b: c \leq a \leq b \leq d$ for which

- $\tilde{u}(x)$ is monotonic increasing on $[c, a]$.

- $\tilde{u}(x)$ is monotonic decreasing on $[b, d]$.

- $\tilde{u}(x)=1, a \leq x \leq b$.

Any real interval number $[a, b]$ can be regarded as a fuzzy number with membership function $\chi_{[a, b]}$. So is any real number $a$ with membership function $\chi_{\{a\}}$. The membership of fuzzy number zero $\hat{0}$ is defined by $\chi_{\{0\}}$. The set of all the fuzzy numbers is denoted by $\mathfrak{E}$. The set of all real numbers $\mathbb{R}$ is actually a proper subset of $\mathfrak{E}$. An equivalent parametric definition is given in (Wu,1991,p33-38) as: A fuzzy number $\tilde{u}$ is an ordered pair of functions $(\underline{u}(r), \bar{u}(r)), 0 \leq r \leq 1$, which satisfy the following requirements: 
- $\underline{u}(r)$ is a bounded left continuous non-decreasing function over $[0,1]$.

- $\bar{u}(r)$ is a bounded left continuous non-increasing function over $[0,1]$.

- $\underline{u}(r) \leq \bar{u}(r), 0 \leq r \leq 1$.

Sets $\{x \in \mathbb{R} \mid \tilde{u}(x)>0\}$ and $\{x \in \mathbb{R} \mid \tilde{u}(x)=1\}$ are called the support and kernel of fuzzy number $\tilde{u}$, denoted by suppũ and ker $\tilde{u}$, respectively. It follows from Definition 1 that ker $\tilde{u} \subset \operatorname{supp} \tilde{u}$ and hence ker $\tilde{u}$ is compact. The middle point of ker $\tilde{u}$ is called the center of $\tilde{u}$. The addition and scalar multiplication of fuzzy numbers in $\mathfrak{E}$ are defined as usual (Frideman, 2006, p1257-1275).

A n-dimensional fuzzy vector is a column vector that is composed of $n$ fuzzy numbers. In particular, each entry of zero fuzzy vector $\widehat{0}$ is the fuzzy number zero $\hat{0}$. Denote $\mathfrak{E}^{n}$ the family of all $n$-dimensional fuzzy number vectors. Define the addition and scalar multiplication of fuzzy vectors as those of real vector except the usual addition and multiplication of entries replaced by addition and scalar multiplication for fuzzy numbers, respectively.

Denote $\mathfrak{F}^{n}[0,1]$ the family of all the vector-valued functions mapping from $[0,1]$ into $\mathbb{R}^{n}$.

Definition 2. For a real matrix $A$, if there exist a real number $\lambda$ and a non-zero real vector $x \in \mathbb{R}^{n}$ satisfy

$$
A x=\lambda x,
$$

then we call $\lambda$ the real eigenvalue (associated with real eigenvectors) of $A, x$ the real eigenvector of $A$ corresponding to $\lambda$.

If there exist a real number $\lambda$ and a non-zero fuzzy vector $\tilde{x} \in \mathfrak{E}^{n}$ satisfy (1), then we call $\lambda$ the real eigenvalue associated with fuzzy eigenvectors of matrix $A, \tilde{x}$ the fuzzy eigenvector of $A$ corresponding to $\lambda$.

A real matrix maybe have complex eigenvalues and associated complex eigenvectors. We will only handle real eigenvalues and fuzzy eigenvectors of a real matrix in this work.

Eq.(1) as a dual fuzzy linear system (Frideman, 2006, p1257-1275) can be represented in the form of following functional linear system:

$$
\left\{\begin{array}{l}
\underline{\lambda x_{i}}=\sum_{j=1}^{n} \underline{a_{i j} x_{j}}, \\
\overline{\lambda x_{i}}=\sum_{j=1}^{n} \overline{a_{i j} x_{j}}, i=1, \ldots, n .
\end{array}\right.
$$

where $x_{i}=\left(\underline{x}_{i}(r), \bar{x}_{i}(r)\right)$.

The functional linear system (2) can be rewritten in red-black partitioned vector-matrix form as follows:

$$
\left(\begin{array}{cc}
\lambda I_{n} & 0 \\
0 & \lambda I_{n}
\end{array}\right)\left(\begin{array}{c}
\underline{X} \\
-\bar{X}
\end{array}\right)=\left(\begin{array}{ll}
S_{1} & S_{2} \\
S_{2} & S_{1}
\end{array}\right)\left(\begin{array}{c}
\underline{X} \\
-\bar{X}
\end{array}\right), \quad \text { if } \lambda \geq 0,
$$

or

$$
\left(\begin{array}{cc}
0 & -\lambda I_{n} \\
-\lambda I_{n} & 0
\end{array}\right)\left(\begin{array}{c}
\frac{X}{-\bar{X}}
\end{array}\right)=\left(\begin{array}{cc}
S_{1} & S_{2} \\
S_{2} & S_{1}
\end{array}\right)\left(\begin{array}{c}
\frac{X}{\bar{X}} \\
-\quad \text { if } \lambda<0
\end{array}\right.
$$

where $X=\left(\underline{x}_{1}, \ldots, \underline{x}_{n},-\bar{x}_{1}, \ldots,-\bar{x}_{n}\right)^{T}=\left(\underline{X}^{T},-\bar{X}^{T}\right)^{T}$ and the entries of matrix $S=\left(\begin{array}{cc}S_{1} & S_{2} \\ S_{2} & S_{1}\end{array}\right)$ are defined as follows:

$$
\begin{aligned}
& a_{i j} \geq 0 \Rightarrow s_{i j}=s_{i+n, j+n}=a_{i j}, \\
& a_{i j}<0 \Rightarrow s_{i+n, j}=s_{i, j+n}=-a_{i j}
\end{aligned}
$$

and any $s_{i j}$ which is not determined is zero such that $A=S_{1}-S_{2}$.

The $n \times n$ matrix $S_{1}$ contains the nonnegative entries of $A$ while $S_{2}$ is made of the absolute values of negative entries of $A$ and $A=S_{1}-S_{2}, S_{1}+S_{2}=|A|=\left(\left|a_{i j}\right|\right)_{n \times n}$. Throughout this paper, $|M|_{m \times n}$ denotes the nonnegative matrix $\left(\left|m_{i j}\right|\right)_{m \times n}$, where $M=\left(m_{i j}\right)_{m \times n}$.

\section{Fuzzy eigenvectors of real matrix}

\subsection{Eigenvalues associated with fuzzy eigenvectors of real matrix}

Theorem 1. A real number $\lambda$ and a non-zero fuzzy vector $\tilde{x}$ satisfy (1) only if $\lambda$ is one of eigenvalues of matrix $A$ or one of the positive eigenvalues of $|A|$ or one of the opposite numbers of positive eigenvalues of $|A|$.

Proof: When $\lambda$ is one of eigenvalues of $A$, it is obvious that the result holds. Next we suppose $\operatorname{that} \operatorname{det}\left(\lambda I_{n}-A\right) \neq 0$.

The real number $\lambda$ and non-zero fuzzy vector $\tilde{x}=\left(\tilde{x}_{1}, \cdots, \tilde{x}_{n}\right)^{T}$ satisfy (1) only if $\lambda$ and non-zero functional vector $X=\left(\underline{x}_{1}, \cdots, \underline{x}_{n},-\bar{x}_{1}, \cdots,-\bar{x}_{n}\right)^{T} \in \mathfrak{F}^{2 n}[0,1]$ satisfy (3) or (4). 
If $\lambda>0$, the system of linear equations (3) in $\mathfrak{F}^{2 n}[0,1]$ has non-zero solutions only if $\operatorname{det}\left(\lambda I_{2 n}-S\right)=0$. A simple calculation shows that $\operatorname{det}\left(\lambda I_{2 n}-S\right)=\operatorname{det}\left(\lambda I_{n}-A\right) \operatorname{det}\left(\lambda I_{n}-|A|\right)$. Then $\lambda$ is a positive eigenvalue of $|A|$.

If $\lambda=0$ is an eigenvalue of $|A|$ but not of $A$, then $A x=\widehat{0}$ has only trivial solution due to Lemma 2 .

If $\lambda<0$, the system of linear equations (4) in $\mathfrak{F}^{2 n}[0,1]$ has non-zero solutions only if

$$
\operatorname{det}\left(\begin{array}{cc}
-S_{1} & -\lambda I_{n}-S_{2} \\
-\lambda I_{n}-S_{2} & -S_{1}
\end{array}\right)=0 .
$$

Same calculation gives $\operatorname{det}\left(\begin{array}{cc}-S_{1} & -\lambda I_{n}-S_{2} \\ -\lambda I_{n}-S_{2} & -S_{1}\end{array}\right)=\operatorname{det}\left(-\lambda I_{n}-|A|\right) \operatorname{det}\left(\lambda I_{n}-A\right)$. Hence $\lambda$ is one of the opposite number of positive eigenvalues of $|A|$.

It is strange to find that the positive eigenvalues of $|A|$ or their opposite numbers may also be or not be the real eigenvalues associated with fuzzy eigenvectors of $A$. The below examples show that these cases actually happen. For matrix $\left(\begin{array}{ll}1 & 2 \\ 1 & -2\end{array}\right)$, 3 is not its eigenvalue associated with real eigenvectors. However, it is easy to check that $\left(\begin{array}{cc}1 & 2 \\ 1 & -2\end{array}\right) \tilde{u}= \pm 3 \tilde{u}$ with $\tilde{u}=$ $\left(\begin{array}{cc}(0.5 r-0.5 & 0.5-0.5 r) \\ (0.5 r-0.5 & 0.5-0.5 r)\end{array}\right)$. This means that $\tilde{u}$ is a fuzzy eigenvector corresponding to the eigenvalue \pm 3 of this matrix.

For another real matrix $A=\left(\begin{array}{cc}-5 & 2 \\ 1 & 4\end{array}\right), \lambda_{1,2}=\frac{-1 \pm \sqrt{89}}{2}$ are two eigenvalues of $A$ and 3,6 are those of $|A|$. It is not hard to prove that $A \tilde{x}= \pm 6 \tilde{x}$ has nontrivial solution in $\mathfrak{E}^{n}$ and $A \tilde{x}= \pm 3 \tilde{x}$ has not.

\subsection{Structure of fuzzy eigenvectors}

Theorem 2. If there exist a real number $\lambda$ and a non-zero fuzzy vector $\tilde{x}$ satisfy (1) and $\lambda$ is one of the positive eigenvalues of $|A|$ or its opposite number but not eigenvalue of $A$ then $\tilde{x}$ is a symmetric fuzzy number with 0 being its center.

Proof: If $\lambda>0$ and $\operatorname{det}\left(\lambda I_{n}-A\right) \neq 0, \operatorname{det}\left(\lambda I_{n}-|A|\right)=0$ then (3) is equivalent to

$$
\left\{\begin{array}{l}
S_{1} \underline{X}-\lambda \underline{X}-S_{2} \bar{X}=0 \\
S_{2} \underline{X}-S_{1} \bar{X}+\lambda \bar{X}=0
\end{array}\right.
$$

Thus $\left(A-\lambda I_{n}\right)(\underline{X}+\bar{X})=0,\left(|A|-\lambda I_{n}\right)(\underline{X}-\bar{X})=0$. So, $\underline{X}=-\bar{X}$. Because $\tilde{x} \in \mathfrak{E}^{n}, \underline{X}(r)$ is monotonously increasing on $[0,1]$ and $\underline{X}(r) \leq \bar{X}(r)$ for all $r \in[0,1]$. It follows that each entry of $\tilde{X}$ is a symmetric fuzzy number with 0 being its center.

If $\lambda<0$ and $\operatorname{det}\left(\lambda I_{n}-A\right) \neq 0, \operatorname{det}\left(\lambda I_{n}-|A|\right)=0$ then (4) is equivalent to

$$
\left\{\begin{array}{l}
S_{1} \underline{X}-\lambda \bar{X}-S_{2} \bar{X}=0 \\
S_{2} \underline{X}-S_{1} \bar{X}+\lambda \underline{X}=0
\end{array}\right.
$$

The result follows similar argument as the preceding case $\lambda>0$.

Theorem 3. If there exist a real number $\lambda$ and a non-zero fuzzy vector $\tilde{x}$ satisfy (1) and $\lambda$ is one of eigenvalues of $A$ but not of $|A|$ then $\tilde{x} \in \mathbb{R}^{n}$.

Proof: If $\lambda \geq 0$ and $\operatorname{det}\left(\lambda I_{n}-A\right) 0, \operatorname{det}\left(\lambda I_{n}-|A|\right) \neq 0$ then it follows from (5) that $\left(|A|-\lambda I_{n}\right)(\underline{X}-\bar{X})=0$. Thus, $\underline{X}=\bar{X}$. That is $\underline{X}(r)=\bar{X}(r)$ for all $r \in[0,1]$. However, $\underline{X}(r)$ and $\bar{X}(r)$ are monotonously increasing and decreasing on [0,1], respectively. It follows that $\underline{X}(r)=\bar{X}(r)=\underline{X}(1) \in \mathbb{R}^{n}$, i.e. $x \in \mathbb{R}^{n}$.

If $\lambda<0$ and $\operatorname{det}\left(\lambda I_{n}-A\right)=0, \operatorname{det}\left(-\lambda I_{n}-|A|\right) \neq 0$ then it follows from (6) that $\left(|A|-\lambda I_{n}\right)(\underline{X}-\bar{X})=0$. Thus, $\underline{X}=\bar{X}$. The result follows similar argument as the above case.

Examples in subsection 3.1 explore that when $\lambda$ is not an eigenvalue of $A$ but one of the positive eigenvalues of $|A|$ or their opposite numbers, then it maybe satisfy (1) or not. We have to choose those who make Eq.(1) holds.

Theorem 4. If $\lambda>0$ is an eigenvalue of $|A|$ but not of $A$, then there exist a nonzero fuzzy vector $\tilde{x}$ satisfy Eq.(1) if and only if $(|A|-\lambda I) \alpha=0$ has nontrivial nonnegative solution in $\mathbb{R}^{n}$.

If $\lambda<0$ is the opposite number of an eigenvalue of $|A|$ but not an eigenvalue of $A$, then there exist a nonzero fuzzy vector $\tilde{x}$ satisfy Eq.(1) if and only if $(|A|+\lambda I) \alpha=0$ has nontrivial nonnegative solution in $\mathbb{R}^{n}$.

Proof: If $\lambda>0$ and $\operatorname{det}\left(\lambda I_{n}-A\right) \neq 0, \operatorname{det}\left(\lambda I_{n}-|A|\right)=0$ then Eq.(1) is equivalent to the following linear system holds

$$
\left\{\begin{aligned}
& \underline{X}=-\bar{X} \\
\left(|A|-\lambda I_{n}\right) \underline{X} & =0
\end{aligned}\right.
$$

and $\underline{X}(r)$ is monotonously increasing on [0,1], moreover, $\underline{X}(r) \leq \bar{X}(r)$ for all $r \in[0,1]$. It follows from Theorem 3 in (Tian, 2008, p161-165) that if fuzzy linear system $A \tilde{x}=\tilde{y}$ has a non-triangular fuzzy number solution then it must have a triangular fuzzy number solution, where $\tilde{x}, \tilde{y} \in \mathfrak{E}^{n}$ and $A$ is a crisp real matrix. According to Theorem 2 , let 
$\underline{X}(r)=-\alpha+\alpha r$, where $\alpha \in \mathbb{R}^{n}$. It follows that when $\lambda>0$, there exist a nontrivial $\tilde{x} \in \mathfrak{E}^{n}$ such that Eq.(1) holds if and only if $(|A|-\lambda I) \alpha=0$ has nontrivial nonnegative solution in $\mathbb{R}^{n}$.

If $\lambda<0$ and $\operatorname{det}\left(\lambda I_{n}-A\right) \neq 0, \operatorname{det}\left(-\lambda I_{n}-|A|\right)=0$ then Eq.(1) is equivalent to

$$
\left\{\begin{aligned}
\underline{X} & =-\bar{X} \\
\left(|A|+\lambda I_{n}\right) \underline{X} & =0
\end{aligned}\right.
$$

and $\underline{X}(r), \bar{X}(r)$ satisfy the same conditions as the above case. Using the similar argument as case $\lambda>0$ yields the conclusion.

\section{The structure of fuzzy eigenspaces}

Denote $V_{\lambda}^{F}$ and $V_{\lambda}^{R}$ the families of all fuzzy and real eigenvectors of matrix $A$ corresponding to eigenvalue $\lambda$, and call them fuzzy and real eigenspaces of matrix $A$, respectively.

The next theorem shows that fuzzy eigenspace as a subspace of $\mathfrak{E}^{n}$ is closed under addition and scalar multiplication.

Theorem 5. $V_{\lambda}^{F}$ is closed under addition and scalar multiplication.

Proof: Let $\tilde{u}, \tilde{v} \in V_{\lambda}^{F}$, then $A \tilde{u}=\lambda \tilde{u}$ and $A \tilde{v}=\lambda \tilde{v}$ with $\tilde{u}, \tilde{v} \in \mathfrak{E}^{n}$. The $i$-th entry of $A(\tilde{u}+\tilde{v}) \operatorname{satisfies}[A(\tilde{u}+\tilde{v})]_{i}=$ $\sum_{j=1}^{n} a_{i j}\left(\tilde{u}_{j}+\tilde{v}_{j}\right)=\sum_{j=1}^{n}\left(a_{i j} \tilde{u}_{j}+a_{i j} \tilde{v}_{j}\right)=\sum_{j=1}^{n} a_{i j} \tilde{u}_{j}+\sum_{j=1}^{n} a_{i j} \tilde{v}_{j}=(A \tilde{u})_{i}+(A \tilde{v})_{i}$. Hence $A(\tilde{u}+\tilde{v})=A \tilde{u}+A \tilde{v}=\lambda \tilde{u}+\lambda \tilde{v}=\lambda(\tilde{u}+\tilde{v})$.

Let $k \in \mathbb{R}$, then the $i$-th entry of $A(k \tilde{u})$ satisfies

$$
[A(k \tilde{u})]_{i}=\sum_{j=1}^{n} a_{i j}\left(k \tilde{u}_{j}\right)=\sum_{j=1}^{n}\left(k a_{i j}\right) \tilde{u}_{j}=k \sum_{j=1}^{n} a_{i j} \tilde{u}_{j}=k(A \tilde{u})_{i} .
$$

Hence $A(k \tilde{u})=k A \tilde{u}=k \lambda \tilde{u}=\lambda(k \tilde{u})$.

The following theorem tells us the fuzzy eigenspace is natural extension of the real eigenspace of a real matrix.

Theorem 6. Linear space $V_{\lambda}^{R}$ is a subset of $V_{\lambda}^{F}$.

Proof: Take arbitrary $x \in V_{\lambda}^{R}$. Then $x=\left(x_{1}, \cdots, x_{n}\right)^{T} \in \mathbb{R}^{n}$ and $A x=\lambda x$. Construct fuzzy vector $\tilde{x}=\left(\tilde{x}_{1}, \cdots, \tilde{x}_{n}\right)^{T}$ in which $\tilde{x}_{i}=\left(\underline{x}_{i}(r), \bar{x}_{i}(r)\right)$ with $\underline{x}_{i}(r)=\bar{x}_{i}(r)=x_{i}$, for all $r \in[0,1]$.

Let $S=\left(\begin{array}{ll}S_{1} & S_{2} \\ S_{2} & S_{1}\end{array}\right)$ and $X=\left(\underline{x}_{1}, \cdots, \underline{x}_{n},-\bar{x}_{1}, \cdots,-\bar{x}_{n}\right)^{T}=\left(x^{T},-x^{T}\right)^{T}$. So

$$
S X=\left(\begin{array}{ll}
S_{1} & S_{2} \\
S_{2} & S_{1}
\end{array}\right)\left(\begin{array}{c}
x \\
-x
\end{array}\right)=\left(\begin{array}{c}
A x \\
-A x
\end{array}\right)=\left(\begin{array}{c}
\lambda x \\
-\lambda x
\end{array}\right)=\lambda\left(\begin{array}{c}
x \\
-x
\end{array}\right)=\lambda X
$$

Hence $\tilde{x}$ is a fuzzy eigenvector of $A$ corresponding to $\lambda$, i.e. $\tilde{x} \in V_{\lambda}^{F}$. However, since the $i$-th entry of $\tilde{x}$ is actually real number $x_{i}, \tilde{x}=x$ and then $V_{\lambda}^{R} \subset V_{\lambda}^{F}$.

The intersection of two fuzzy eigenspaces of a real matrix corresponding to two different eigenvalues is zero space as below theorem shows.

Theorem 7. If $\lambda_{1}$ and $\lambda_{2}$ are two different eigenvalues of $A$, then $V_{\lambda_{1}}^{F} \cap V_{\lambda_{2}}^{F}=\{0\}$.

Proof: Let $\tilde{u} \in V_{\lambda_{1}}^{F} \cap V_{\lambda_{2}}^{F}$, then $A \tilde{u}=\lambda_{i} \tilde{u}, i=1,2$, i.e. $S U=\lambda_{i} U$ or $\left(\begin{array}{cc}S_{1} & S_{2} \\ S_{2} & S_{1}\end{array}\right)\left(\begin{array}{c}\frac{U}{-}(r) \\ -\bar{U}(r)\end{array}\right)=\lambda_{i}\left(\begin{array}{c}\tilde{u}(r) \\ -\tilde{u}(r)\end{array}\right)$. This means that $\left(\lambda_{1}-\lambda_{2}\right)\left(\begin{array}{c}\underline{U}(r) \\ -\bar{U}(r)\end{array}\right)=0$. So $\underline{U}(r)=\bar{U}(r)=0$ for all $r \in[0,1]$ since $\lambda_{1} \neq \lambda_{2}$.

Theorem 8. If $\lambda=0$ is an eigenvalue of $A$ and $A$ has no column which consists of zeros except the $i$-th one, then $V_{0}^{F}$ is the family of all fuzzy vectors $\left(u_{1}, \ldots, \tilde{u}_{i}, \ldots, u_{n}\right)^{T}$, where $\tilde{u}_{i} \in \mathfrak{E}$ and $\left(u_{1}, \ldots, u_{i-1}, u_{i+1}, \ldots, u_{n}\right)^{T}$ is the real solution vector of $B x=\hat{0}$ where $B$ is the matrix obtained by deleting the $i$-th column from $A$.

Proof: Let $\tilde{u}=\left(\tilde{u}_{1}, \cdots, \tilde{u}_{n}\right)^{T}$ be an arbitrary fuzzy vector in $V_{0}^{F}$, then $\tilde{u} \in \mathfrak{E}^{n}$ and $A \tilde{u}=0 \tilde{u}$. The $k$-th entry of $A \tilde{u}$ satisfies $[A \tilde{u}]_{k}=\sum_{j=1}^{n} a_{k j} \tilde{u}_{k}=\sum_{j=1, k \neq i}^{n} a_{k j} \tilde{u}_{j}+0 \tilde{u}_{i}=0$. Hence $\tilde{u}_{i} \in \mathfrak{E}$ and $\left(u_{1}, \ldots, u_{i-1}, u_{i+1}, \ldots, u_{n}\right)^{T}$ is the real solution vector of $B x=\hat{0}$ from Lemma 2 , where $B$ is a matrix obtained by deleting the $i$-th column from $A$.

Corollary 1. Assume that the $i_{1}$-th,..., $i_{k}$-th columns of $A$ are zero vectors and others not zeros. Denote the matrix obtained by deleting the $i_{1}$-th, .., $i_{k}$-th columns from $A$ by $B$, the solution of fuzzy linear system $B x=\hat{0}$ by $v=\left(u_{\alpha}\right)^{T}$, $\alpha \in\{1, \ldots, n\} \backslash\left\{i_{1}, \ldots, i_{k}\right\}$. Then $\lambda=0$ is an eigenvalue of $A$ and $V_{0}^{F}=\left\{\left(u_{1}, \ldots, \tilde{u}_{i_{1}}, \ldots, \tilde{u}_{i_{k}}, \ldots, u_{n}\right)^{T}\right\}$ with $\tilde{u}_{i_{j}} \in \mathfrak{E}, j=1, \ldots, k$.

\section{Relationship between the fuzzy eigenvectors and real eigenvectors}

A fuzzy number can be defuzzified to a real one by letting its center be the model. Applied similar argument to fuzzy vector, fuzzy vector can be defuzzified to a real one. The next two theorems answer whether a real vector obtained by defuzzified fuzzy eigenvector corresponding to $\lambda$ is still a real eigenvector corresponding to $\lambda$. 
Theorem 9. If $\lambda$ is a positive eigenvalue of $|A|$ but not eigenvalue of $A$, then real vectors defuzzified from those in $V_{\lambda}^{F}$ are all real zero vectors.

Proof: Let $\tilde{u}=\left(\tilde{u}_{1}, \cdots, \tilde{u}_{n}\right)^{T}$ be an arbitrary fuzzy vector in $V_{\lambda}^{F}$. Defuzzify $\tilde{u}$ to $u^{c}=\left(u_{1}^{c}, \cdots, u_{n}^{c}\right)^{T}$, where $u_{i}^{c}$ is the center of the $i$-th entry of $\tilde{u}$. Then $u^{c} \in \mathbb{R}^{n}$ and $A u^{c}=\lambda u^{c}$, hence $(\lambda I-A) u^{c}=0$. Therefore, $u^{c}=0$ since $\lambda I-A$ is invertible.

Theorem 10. Let $\lambda$ be an eigenvalue of $A$. If real vectors defuzzified from those in $V_{\lambda}^{F}$ are not zeros, then they are all real eigenvectors of $A$ corresponding to $\lambda$.

Proof: The proof is similar to that of Theorem 9 except that $\lambda$ is an eigenvalue of $A$.

The following deals with the case in which 0 is an eigenvalue associated with fuzzy eigenvectors of $A$.

Lemma 2. The homogenous fuzzy linear system $A \tilde{x}=\widehat{0}$ has only real solution vectors if $A$ has no column which consists of zeros (Tian,2008,p161-165).

Corollary 2. If $\lambda=0$ is an eigenvalue associated with fuzzy eigenvectors of real matrix $A$ and $A$ has no column which consists of zeros, then the fuzzy eigenvectors corresponding to $\lambda=0$ are all real vectors, in other words, $V_{0}^{R}=V_{0}^{F}$.

This corollary shows that real eigenvectors corresponding to eigenvalue 0 of $A$ cannot be fuzzified when $A$ has no column which consists of zeros.

\section{An application of fuzzy eigenvector}

When the coefficients matrix $A$ is invertible, the linear system $A x=y, y \in \mathbb{R}^{n}$ has one and only one solution $x^{*}$ in $\mathbb{R}^{n}$. However, wether its fuzzy counterpart $A \tilde{x}=\tilde{y}, \tilde{y} \in \mathfrak{E}^{n}$ has solution in $\mathfrak{E}^{n}$ will depend on the right hand side $\tilde{y}$ even though $A$ is invertible (Frideman, 2006, p1257-1275).

Suppose that $\lambda_{1}, \lambda_{2}, \cdots, \lambda_{m}$ are the real eigenvalues of nonsingular matrix $A$ and $V_{\lambda_{1}}, \cdots, V_{\lambda_{m}}$ are their corresponding eigenspaces of $A$. Denote $\bigoplus V_{\lambda_{i}}=V_{\lambda_{1}}+\cdots+V_{\lambda_{m}} \subset \mathbb{R}^{n}$. It is obvious that $x^{*}=\sum_{i=1}^{m} \frac{k_{i}}{\lambda_{i}} u_{i}$ is the solution in $\mathbb{R}^{n}$ of $A x=y$ when $y=\sum_{i=1}^{m} k_{i} u_{i} \in \bigoplus V_{\lambda_{i}}$. We will prove this result is valid for fuzzy linear system $A \tilde{x}=\tilde{y}, \tilde{y} \in \mathfrak{E}^{n}$, i.e. we give a sufficient condition to existence of strong solution of fuzzy linear system.

Denote $\bigoplus_{\lambda \in \Lambda} V_{\lambda}^{F}(A)$ the collection of all linear combinations of those fuzzy vectors in $V_{\lambda}^{F}$, where $\Lambda=\left\{\lambda_{1}, \ldots, \lambda_{m}\right\}$ is composed of all eigenvalues associated with fuzzy eigenvectors of real matrix $A$.

Theorem 12. $\bigoplus_{\lambda \in \Lambda} V_{\lambda}^{F}(A)$ as a subspace of $\mathfrak{E}^{n}$ is closed under addition and scalar multiplication.

Proof: Let $\tilde{u}, \tilde{v} \in \bigoplus_{\lambda \in \Lambda} V_{\lambda}^{F}(A)$ and $q \in \mathbb{R}$, then there exists real weights $k_{i}, l_{i}, i \in\{1, \ldots, m\}$ such that $\tilde{u}=\sum_{i=1}^{m} k_{i} \tilde{u}_{i}, \tilde{v}=$ $\sum_{i=1}^{m} l_{i} \tilde{u}_{i}^{\prime}$ where $\tilde{u}_{i}, \tilde{u}_{i}^{\prime} \in V_{\lambda_{i}}^{F}$. Consider Theorem 5 and let $\tilde{u}_{i}^{\prime \prime}=k_{i} \tilde{u}_{i}+l_{i} \tilde{u}_{i}^{\prime}$ then $\tilde{u}_{i}^{\prime \prime},\left(q k_{i}\right) \tilde{u}_{i} \in V_{\lambda_{i}}^{F}$. Hence $\tilde{u}+\tilde{v}=\sum_{i=1}^{m} k_{i} \tilde{u}_{i}+$ $\sum_{i=1}^{m} l_{i} \tilde{u}_{i}^{\prime}=\sum_{i=1}^{m}\left(k_{i} \tilde{u}_{i}+l_{i} \tilde{u}_{i}^{\prime}\right)=\sum_{i=1}^{m} \tilde{u}_{i}^{\prime \prime} \in \bigoplus_{\lambda \in \Lambda} V_{\lambda}^{F}(A)$ and $q \tilde{u}=q \sum_{i=1}^{m} k_{i} \tilde{u}_{i}=\sum_{i=1}^{m}\left(q k_{i}\right) \tilde{u}_{i} \in \bigoplus_{\lambda \in \Lambda} V_{\lambda}^{F}(A)$.

Theorem 13. If $A$ is invertible and $\tilde{y} \in \bigoplus_{\lambda \in \Lambda} V_{\lambda}^{F}(A)$, then fuzzy linear system $A \tilde{x}=\tilde{y}, \tilde{y} \in \mathfrak{E}^{n}$ has a solution vector.

Proof: If $\tilde{y} \in \bigoplus_{\lambda \in \Lambda} V_{\lambda}^{F}(A)$ then there exist weights $k_{i}$ and $\tilde{u}_{i} \in V_{\lambda_{i}}^{F}, i \in\{1, \ldots, m\}$ such that $\tilde{y}=\sum_{i=1}^{m} k_{i} \tilde{u}_{i}$. Let $x^{*}=\sum_{i=1}^{m} \frac{k_{i}}{\lambda_{i}} \tilde{u}_{i}$ then $x^{*} \in \mathfrak{E}^{n}$ and

$$
A x^{*}=A \sum_{i=1}^{m} \frac{k_{i}}{\lambda_{i}} \tilde{u}_{i}=\sum_{i=1}^{m} \frac{k_{i}}{\lambda_{i}} A \tilde{u}_{i}=\sum_{i=1}^{m} \frac{k_{i}}{\lambda_{i}} \lambda_{i} \tilde{u}_{i}=\sum_{i=1}^{m} k_{i} \tilde{u}_{i}=\tilde{y} .
$$

This means that $x^{*}$ is a solution vector of $A \tilde{x}=\tilde{y}$.

Corollary 3. Fuzzy linear system $A \tilde{x}=\tilde{y}$ has solution vector if $\tilde{y}=\sum_{i=1}^{m} k_{i} \tilde{u}_{i} \in \bigoplus_{\lambda \in \Lambda} V_{\lambda}^{F}(A)$, where $k_{j}=0$ when $\lambda_{j}=0$.

\section{Conclusion}

In this work, we investigate fuzzy eigenvectors, fuzzy eigenspaces of real matrix and the relationships between real eigenspace and fuzzy eigenspace. As an application of fuzzy eigenvector, we use fuzzy eigenvector to give a sufficient condition to existence of solution vector of fuzzy linear system $A \tilde{x}=\tilde{y}$, where $A$ is a crisp square matrix, $\tilde{x}$ and $\tilde{y}$ are fuzzy vectors.

\section{References}

Alevizos P. Theodorou Y. \& Drossos C. (2007). Correspondence analysis with fuzzy data: The fuzzy eigenvalue problem. Fuzzy sets and systems, 158, 704-721.

Chin Kwai-Sang \& Wang Ying-Ming. (2006). An eigenvector method for generating normalized interval and fuzzy weights. Applied Mathematics and Computation,181,1257-1275.

D. Dubois, H. Prade(Eds.). (1980). Fuzzy Sets and Systems: Theory and Application. New York: Academic Press.

Horn R.A. \& Johnson C. R.(Ed.). (1985). Matrix analysis. England: Cambridge Press.

J.J. Buckley. (1990). Fuzzy eigenvalues and input-output analysis. Fuzzy Sets and Systems, 34, 187-195. 
M. Frideman, et al.(1998). Fuzzy linear systems. Fuzzy Sets and Systems, 96, 201-209.

Ma M., et al. (2000). Duality in Fuzzy linear systems. Fuzzy Sets and Systems, 109, 55-58.

Massa F., et al. (2008). A complete method for efficient fuzzy modal analysis. Journal of Sound and Vibration,309, 63-85.

TIAN Zengfeng \& HU Liangjian. (2008). The structure of solution of fuzzy linear system (in Chinese). Basic Sciences Journal of Textile Universities, 2,161-165.

Wang G., Li Y. \& Wen C.(2007). On fuzzy n-cell numbers and n-dimension fuzzy vectors. Fuzzy Sets and Systems,158, $71-84$.

Wang K. \& Zheng B. (2006). Symmetric successive overrelaxation methods for fuzzy linear systems. Applied Mathematics and Computation, 175, 891-901.

Wang X., Zhong Z. \& Ha M. (2001). Iteration algorithm for solving a system of fuzzy linear equations. Fuzzy Sets and Systems, 119,121-128.

Wu Cong-Xin \& Ma Ming. (1991). Embedding problem of fuzzy number space:Part I. Fuzzy Sets and Systems,44,33-38. Zadeh L. A. (1965). Fuzzy Sets. Information Control,8,338-353. 